\section{Recall load in STM*}

\author{
GEORGE E. STELMACH and JACK R. BRUCE $\dagger$ \\ University of California, Santa Barbara, Calif. 93106
}

Recall load and difficulty of information processing was examined in kinesthetic recall using a within-S design $(\mathrm{N}=15)$. The multiple-recall task had larger absolute error compared to the single task; however, algebraic error revealed little differences. Absolute error analysis indicated that both retention interval conditions were significantly different from control. Difficulty of information reduction activity was not found to be a significant variable, and the Recall Load by Interpolated Activity interaction failed significance. No evidence was found to suggest that relative difficulty between the motor and verbal task can explain the empirical differences between the two domains.

In one of the first investigations on motor short-term memory (STM), Adams \& Dijkstra (1966) found that retention of simple linear movements were subject to rapid forgetting over short periods of time and that increasing the number of reinforcements reduced the retention loss. These findings suggested that motor retention might follow the same basic laws observed in verbal STM. These facts have led investigators into many facets of short-term motor retention (Posner, 1967; Stelmach, 1969; Pepper \& Herman, 1970). As a result, the difference between verbal and motor STM is gradually emerging. The main empirical difference appears to be centered on the effect interpolated activity has on retention.

In verbal behavior, forgetting occurs only when retention intervals are filled with some interpolated task (Peterson \& Peterson, 1959; Posner \& Rossman, 1965). In addition, similarity between original and interpolated items and available channel processing capacity augments short-term forgetting (Wickelgren, 1965; Posner \& Konick, 1966; Posner \& Rossman, 1965).

In contrast, short-term kinesthetic retention decreases spontaneously regardless of whether the retention interval is filled (Posner \& Konick, 1966; Pepper \& Herman, 1970). Neither similarity of responses nor difficulty of information transformation have been found to alter the course of forgetting (Posner, 1967; Williams, Beaver, Spence, \& Rundell, 1969). Yet, these same studies have revealed that interpolated activity acted primarily to augment the memory trace, thereby causing recall enors to be shifted in a positive direction (Pepper \& Herman, 1970). The foregoing results have discouraged some investigators from pursuing the development of a unitary theory for verbal and motor STM.

*This study was supported by funds from the Research Committee, University of California. Santa Barbara.

$\div$ Now at the University of Guclph. Ontario. Canada. of the relative difficulty between the two
Why should forgetting in these two types of behavior exhibit different recall characteristics? One possible reason, suggested by Williams et al (1969), is that types of tasks commonly used in motor and verbal STM studies. They have suggested that discrete kinesthetic responses are too simple compared to the verbal STM items, and that making the kinesthetic task more demanding might generate forgetting effects comparable to those of verbal STM.

One way of making the kinesthetic task more demanding during storage, retrieval, and recall is to increase the number of target locations to which $S$ must attend. However, if one used multiple responses, he could introduce a larger verbal component to the kinesthetic memory task since $S$ may use a verbal label to order the presentation and recall of the multiple targets serially. If the stored trace becomes primarily verbal in nature, the recall data might make motor forgetting appear comparable to verbal forgetting. The spontaneous forgetting of kinesthetic responses over unfilled retention intervals makes this possibility seem unlikely (Adams \& Dijkstra, 1966). Moreover, if one recognizes this risk, and, indeed, the empirical facts for the multiple response condition are in close agreement with verbal STM, one can set out to partial out this possible confounding systematically by manipulating the sequence of motor and verbal items. The present report describes an experiment which examined whether increasing S's kinesthetic recall load would cause difficulty of information processing

Table 1

Means and Variances of Algebraic Errors in Degrees for the Two Kinesthetic Tasks

\begin{tabular}{|c|c|c|c|c|c|c|}
\hline \multirow{3}{*}{$\begin{array}{l}\text { Retention } \\
\text { Interval } \\
\text { Condition }\end{array}$} & \multirow{2}{*}{\multicolumn{2}{|c|}{ Single Target Task }} & \multicolumn{4}{|c|}{ Multiple Target Task } \\
\hline & & & \multicolumn{2}{|c|}{ First Target Only } & \multicolumn{2}{|c|}{ Three Target Averag } \\
\hline & Mean & Variance & Mean & Variance & Mean & Variance \\
\hline Add & +2.7 & 23.6 & +1.9 & 19.1 & +1.8 & 9.3 \\
\hline Mimic & +0.1 & 17.9 & +0.3 & 28.6 & +0.3 & 12.6 \\
\hline Rest & -1.9 & 14.0 & -0.1 & 20.2 & +0.4 & 12.2 \\
\hline
\end{tabular}

to be a potent variable in motor STM. SUBJECTS

Fifteen righthanded male and female students, aged 18.22, served as Ss. APPARATUS

The apparatus used consisted of a free-moving manual lever. The lever was similar to one described in an earlier paper (Stelmach, 1969). The only mechanical difference was that this lever handle was longer. For this lever, $1 \mathrm{deg}$ of displacement equaled $4.2 \mathrm{~mm}$. At the start of each trial, the lever was set at a 10-deg starting position. All retention intervals, advancement of slides, and sequences of events were controlled by a digital logic system in conjunction with a tape recorder. PROCEDURE

Each $S$ was told he would receive two blocks of six trials during the experimental session. In one block of trials $S$ recalled only a single target location. For the remaining block, $S$ recalled multiple target locations.In the former condition $S$ received the command "MOVE," and he displaced the lever in a counterclockwise manner until it struck a stop peg which defined a target location. After remaining at the target $2 \mathrm{sec}, S$ heard the command "RETURN," at which point he returned the lever to the starting position, and the retention interval began. Twenty seconds later, with the stop peg removed, S received the command "ESTIMATE," and he attempted to recall the target location by displacing the lever to the correct position. Recall scores were recorded as deviation from the target in degree. For this condition, six target locations were used $(30,50,60,105,125$, and $175 \mathrm{deg}$ from horizontal).

In the multiple recall condition, $S$ was given the command "MOVE" three times. Each time S heard the command "MOVE," he moved the lever to another target location. On the first command, $S$ displaced the lever to the left until it struck a stop peg which defined one of the three target locations. As $S$ moved to the first target location, $E$ inserted the stop peg for the second target location. Two seconds after $S$ reached the first location, $S$ received the second command "MOVE." The lever was then displaced to the right until it struck the peg at the second target location, and $E$ removed the peg which 
defined Target 1. Two seconds later the command "MOVE" was repeated and S moved the lever to the left until it struck another peg. Two seconds after reaching the third location, $S$ received the command "RETURN," and he returned the lever to the starting position, and the retention interval began. Thus, recall of the target-location combinations was always in a left-right-left sequence. At the end of the $20-\mathrm{sec}$ retention interval, $S$ recalled the target positions in the order of presentation with all stop pegs removed. For the multiple-recall condition, six target-location combinations were used $(30,20,50 \mathrm{deg} ; 45,25,75 \mathrm{deg} ; 80,70$, $90 \mathrm{deg} ; 130,80,110 \mathrm{deg} ; 145,100$, $130 \mathrm{deg} ; 165,150,170 \mathrm{deg}$ ).

Both the single- and multiple-recall conditions were administered under control, mimic, and add conditions. In control, $S$ sat passively during the retention interval and waited for the command "ESTIMATE." The mimic condition required $S$ to duplicate pairs of angles shown on a screen by displacing the lever actively. At the onset of the retention interval, $\mathrm{S}$ moved the lever to vertical and began mimicking the pairs of angles in a left-right order, always returning to vertical after completing the two movements. The angles were presented by a Kodak 800 Carousel projector. The slides were advanced at a rate of one per $5 \mathrm{sec}$, with four slides being presented during the retention interval. The complete details of the procedure and the angles used have been described elsewhere (Stelmach, 1970)' The add condition was similar to the foregoing except that after mimicking each angle, $S$ made an additional response to the left which was the sum of the pair of angles. The add condition had an information reduction of 1.86 bits per slide.

All Ss were tested on single- and multiple-recall tasks. The order of testing for the two recall tasks was alternated for every $S$. Retention-interval conditions (control, mimic, and add) were systematically rotated in a balanced order across Ss. The experimental conditions, recall load and interpolated activity, were within-S variables. Target locations and target-location combinations were assigned randomly for each $S$. Throughout the entire session, there was a $30-\mathrm{sec}$ intertrial interval and a 1-min interval between blocks.

\section{RESULTS}

For each trial the difference between the criterion-target location and S's recall estimation was measured in degrees and used as his retention score. In the multiple-response condition, the deviations from the target on the first recall estimation was used as S's retention score for a given trial. This error score is preferable to average multiple-recall error because it avoids serial-response dependence. These data were submitted to both absolute and algebraic analysis.

The mean absolute errors for three retention-interval conditions on the single-target task were $6.8,6.1$, and $5.5 \mathrm{deg}$ for add, mimic, and control, respectively. The corresponding errors for the multiple-recall condition are generally larger. Using these scores, the main effect of recall load was significant, $F(1,14)=4.61, p<.05$. Table 1 gives the mean algebraic errors and their variances for the two recall tasks. Analysis of these means failed to support the foregoing difference between recall loads, $F(1,14)=2.34, p>.05$. However, the variance for the multiple-recall task is slightly larger than for the single-target task.

Inspection of the absolute error reveals that interpolated activity increases the magnitude of the absolute errors. Analysis of this effect indicated that interpolated activity was significant, $F(2,28)=3.62$, $p<.05$. Subsequent Newman-Keuls procedures revealed that both add and mimic conditions were significantly larger than control $(p<.05)$. The difference between add and mimic failed significance.

It can be seen in Table 1 that there is a substantial difference between interpolated activity conditions. With interpolated information processing, the mean errors are relatively large and positive compared to the other two retention-interval conditions. The magnitude of the directional error decreases systematically for the mimic and control conditions, respectively. Statistical evaluation confirms this directional shifting, $F(2,28)=4.10, \quad p<.05$. Newman-Keuls procedures revealed that only interpolated information processing was significantly different from control. It is easily seen in Table 1 that the variances are quite large for all conditions. These large variances make the directional shifting of the means difficult to interpret, since strong response sets do not exist. For both types of analysis, absolute and constant error, the Recall Load by Retention Interval interaction failed significance.

It was hypothesized that increased recall 1 oad would occupy S's information-processing mechanjsm, thereby making available channel-processing capacity an important factor in motor STM. The results did not establish clearly whether increased recall load made the kinesthetic task more demanding. Absolute error did support this interpretation, as the multiple-recall task did have larger error. However, this analysis alone is equivocal since changes in absolute error can be caused by changes in algebraic error or variable error, or both. Even though there was some evidence of directional shifting at recall, algebraic analysis revealed that the tasks were not significantly different. In addition, on the average, the algebraic variance was slightly less for the single-target task. The foregoing results indicate that the increased recall load made $\mathrm{S}$ more variable in his recall estimations.

An additional analysis was made on the two tasks by comparing the three-target average to that of the single target. For both types of error only the main effect of interpolated activity was significant. As can be seen in Table 1, the three-target average has consistently smaller variance, as would be expected due to the method of calculation.

The failure to find a significant Interpolated Activity by Recall Load interaction indicates that there was not a differential effect between information processing and recall load. For mimic and add conditions to be significantly different from control indicates that interpolated activity does affect forgetting. Interpretation of algebraic error suggests that interpolated activity caused $S$ to overestimate the target. However, these means should be interpreted cautiously because of their large variances. Nevertheless, these overshooting trends support Pepper \& Herman's (1970) hypotheses that interpolated activity alters the memory trace. They maintain that the trace is changed by proprioceptive stimulation arising from interpolated tasks. Kinesthetic stimulation from the interpolated act alters the trace by increasing its stored representation. Thus, at recall, $S$ compares the ongoing kinesthetic stimulation with the augmented trace and thereby produces a longer movement. Moreover, this directional shifting also agrees with Posner (1967), Williams et al (1969), and Stelmach (1970).

Nevertheless, Williams et al's (1969) suggestion of relative difficulty between verbal and motor tasks was not supported. However, it should be noted that the multiple-recall task may not have been demanding enough to burden the information processing system.

\section{REFERENCES}

ADAMS, J. A., \& DIJKSTRA, A. Short-term memory for motor responses. Journal of Experimental Psychology, 1966, 71, 314-318. PEPPER, R. L., \& HERMAN, L. M. Decay and interference effects in the short-term retention of a discrete motor act. Journal of 
l cperimental Pstchology Vonosraph Supplement. 1970.83.1-18.

PITERSON. L. R.. \& PITIRSIA. W. J. Short-term retention of individual items. Journal of Lxperimental Psychology. 1959. 58. 193-198.

POSNER. M. I. Characteristics of visual and kinesthetic memory codes. Journal of Experimental Psychology, 1967. 75. 103.107.

POSNER, II. I.. \& KONICK, A. F. Short-term retention of visual and kinesthetic information. Organizational Behavior \& Human Performance, 1966, 1, 71-76.

POSNER, M. I., \& ROSSMAN. E. Effects of size and location of informational transforms upon short-term retention. Journal of Experimental Psycholugy, 1965.70, 496-505.
STEL.MACH. G. L. Prior positioning responses as a factor in short-term retention of a simple motor task. Joumal of Experimental Prycholog 1969.81.523-526.

STEL.MACH. G. E.. \& WTLSON. II Kinesthetic retention. movement extent and information processing. Journal of Experimental Psychology. 1970. in press.

WICKILGREN. W. Acoustic similarity and retroactive interference in short-term memory. Journal of Verbal Learning \& Verbal Behavior, $1965,4,53-61$

WILLIAMS, H. L., BEAVER, W. S., SPENCE, M. T.. \& RCNDELL, O. H. Digital and kinesthetic memory with interpolated information processing. Journal of Experimental Psychology, 1969, 80, 530-536.

\title{
Extinction for systems learning
}

\author{
ADAM MILLER and RUSSELL MCCRIMMON* \\ Saint Cloud State College. Saint Cloud. Minn. 56301
}

With a group procedure, $72 \mathrm{~S} s$ learned associations between pairs of colored figures and nonsense words taken from a 4 by 4 miniature system. In a factorial design, the number of different associations from the system that were learned was varied at two levels, and an extinction procedure that followed learning was applied in three ways. Systems learning occurred. The number of different associations that were learned was not related to accuracy in reproducing the system. For Ss who learned half of the system, the extinction procedure was effective on those associations produced without learning.

One argument for studying artificial linguistic systems is that they demonstrate characteristics of natural languages, e.g., the tendency to regularize inflection of irregular verbs was reproduced by Palermo \& Eberhart (1968). Artificial linguistic systems consist of a set of stimuli and responses arranged so that specific characteristics of the stimuli are systematically associated with particular parts of the responses. An S-R explanation of the observation that, after part of a system was learned, unlearned parts could be produced (Jenkins \& Palermo, 1964), though controversial, offers heuristic focus for investigation of systems learning.

The present study examined the relationships of two variables to systems learning. One concerned an unreplicated relationship reported by Foss (1968) that the larger the number of different associations learned from a system, the more accurate was reproduction of the system. As the second variable, previously unstudied for systems learning, extinction

* Based on a MS thesis at Saint Cloud State College. The current address of the senior author is: Star Route 2, Box 447, Dextcr. Oreg. 97431. was inserted following learning and before reproduction of the system to determine its effect on the learned and unlearned responses of the system.

\section{SUBJECTS}

Seventy-two male and female college students, from 81 volunteers, met the learning criterion (discussed below) and were assigned randomly and evenly among groups.

\section{SYSTEMS LEARNING}

The first experimental step used the miniature system of Foss (1968). It can be represented by a 4 by 4 matrix in which all entries in a single column were the same geometric figure, and each column was a circle, square, triangle, or heart. All entries in a single row were the same color, and each row was red, blue, green, or yellow. Each figure and each color had an associated CVC nonsense syllable for a total of eight CVCs, e.g., ZIN for red and TEP for circle, selected for equal meaningfulness. The possible combinations of colors and shapes produced 16 colored figures and each one had associated with it a six-letter nonsense name, which was the combination of the color CVC and the shape CVC, in that order. For example, the red circle was named ZINTEP. S's task was to learn the names of the colored figures.

The learning materials were sheets of paper partitioned into 16 columns and 16 rows (or 8 by 16 depending on the experimental condition). The columns. headed by the colored figures. and the rows, headed by the nonsense names of figures, were ordered randomly. Each learning sheet was covered by a transparent plastic sheet on which the column and row intersections were covered by black wax that could be scraped away to expose the leaming sheet. Each colored figure had one correct six-letter name associated with it. and their intersection on the learning sheet was marked with the letter "c" (reinforcement). All other intersections were blank. The packet for learning contained five learning sheets in their waxed plastic covers. Each of the five sheets had a different random order of rows and columns.

Ss were instructed to discover and remember the one correct name for each colored figure, but were not told of the systematic relations among CVCs, colors, and shapes. Ss started with the colored figure heading the left-hand column by selecting a name and scraping the wax at the intersection of the row for the name and the left-hand column. At his own pace, $S$ continued until the "c" name was selected for each colored figure in succession on the first learning sheet. After memorizing the associations he had discovered, $S$ placed the first learning sheet in a folder (out of sight) and repeated the procedure for each of the remaining learning sheets until he reached the criterion of selecting the correct nonsense name for each colored figure without error on one sheet. If, on Sheet $5, \mathrm{~S}$ had errors, he was excluded from the experiment.

SYSTEMS EXTINCTION

In the second experimental step, a packet contained five leaming sheets, each 8 by 16 , without "c"s (nonreinforcement), and with new random orders of columns and rows. The procedure varied from learning in that $S$ made only one selection of a nonsense name for each colored figure. Extinction was nonreinforcement following correct responses. Ss were told that the procedure was like learning except that they were to make one choice per figure, could not ask questions, and should complete all five sheets. Ss were not told that there were not "c"s.

\section{SYSTEMS TEST}

Next, Ss completed a systems test, which was a page on which all colored figures and nonsense names of the system formed a 16 by 16 matrix, like that for learning, but with new random orders of columns and rows. $\mathrm{S}$ wrote directly on the 